\title{
Integrated Modeling of Aerosol, Cloud, Precipitation and Land Processes at Satellite- Resolved Scales
}

\author{
Christa Peters-Lidard \\ Chief, Hydrological Sciences Laboratory \\ NASA/GSFC
}

\begin{abstract}
Co-PIs: Wei-Kuo Tao, and Mian Chin
Co-Is: Scott Braun, Jonathan Case, Arthur Hou, Anil Kumar, Sujay Kumar, William Lau, Toshihisa Matsui, Tim Miller, Joseph Santanello, Jr., Jainn Shi, David Starr, Qian Tan, Benjamin Zaitchik
\end{abstract}

In this talk, I will present recent results from a project led at NASA/GSFC, in collaboration with NASA/MSFC and JHU, focused on the development and application of an observation-driven integrated modeling system that represents aerosol, cloud, precipitation and land processes at satellite-resolved scales. The project, known as the NASA Unified WRF (NU-WRF), is funded by NASA's Modeling and Analysis Program, and leverages prior investments from the Air Force Weather Agency and NASA's Earth Science Technology Office (ESTO). We define "satellite-resolved" scales as being within a typical mesoscale atmospheric modeling grid (roughly $1-25 \mathrm{~km}$ ), although this work is designed to bridge the continuum between local (microscale), regional (mesoscale) and global (synoptic) processes. NU-WRF is a superset of the standard NCAR Advanced Research WRF model, achieved by fully integrating the GSFC Land Information System (LIS, already coupled to WRF), the WRF/Chem enabled version of the GOddard Chemistry Aerosols Radiation Transport (GOCART) model, the Goddard Satellite Data Simulation Unit (SDSU), and boundary/initial condition preprocessors for MERRA and GEOS-5 into a single software release (with source code available by agreement with NASA/GSFC). I will show examples where the full coupling between aerosol, cloud, precipitation and land processes is critical for predicting local, regional, and global water and energy cycles, including some high-impact phenomena such as floods, hurricanes, mesoscale convective systems, droughts, and monsoons. 